\title{
Development of a Redox Dye-Based Rapid Colorimetric Assay for the Quantitation of Viability/Mortality of Pine Wilt Nematode
}

\author{
Kyeongmin Han ${ }^{1}$, Jaejoon Lee ${ }^{1}$, Gnanendra Shanmugam ${ }^{1}$, Sun Keun Lee ${ }^{2}$, and Junhyun Jeon ${ }^{1 *}$ \\ ${ }^{1}$ Department of Biotechnology, College of Life and Applied Sciences, Yeungnam University, Gyeongsan 38541, Republic of Korea \\ ${ }^{2}$ Division of Forest Insect Pests and Diseases, National Institute of Forest Science, Seoul 02455, Republic of Korea
}

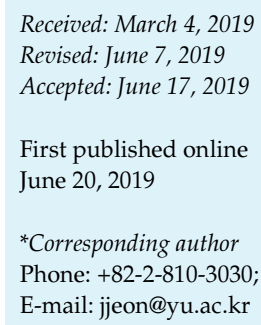

\section{Introduction}

Pine trees, which belong to the pinaceae family, are mainly distributed in the northern hemisphere. Due to various factors, however, the area of pine forests has been decreasing. For example, in the mid-1990s, pine forests accounted for about $60 \%$ of the total forest area of South Korea, but by 2007 , the figure had decreased to $23 \%$. One major constraint that limited pine forests is pine wilt disease (PWD) caused by the insect-vectored pathogen, pine wilt nematode (PWN), Bursaphelenchus xylophilus [1]. PWD was first reported in Japan in 1905 [2] and B. xylophilus was first reported later in the United States in 1934 [3]. Although pine trees in North America are resistant to PWD, the two-leaf pine species in Asia and
Europe are highly susceptible, leading to a major epidemic of the disease [4, 5]. PWD started in Japan, and in the 1980s spread to China, Taiwan and South Korea. In the 1990s, PWD landed in Central and South America and Europe, becoming the most devastating disease in pine trees $[6,7]$.

Such significant impact of PWD stems from the fact that infected trees show nearly $100 \%$ mortality and that there is no definitive control method $[8,9]$. To date, control of the disease has involved control of insect vectors as well as use of chemicals having nematicidal activity. Use of chemicals such as abamectin, though highly effective against $B$. xylophilus, has potential risks to humans and animals [10], in addition to the high amount of labor and cost needed for trunk injection of chemicals.

In line with this, a number of studies have been aimed at 
finding alternative, potential nematicidal compounds that are derived from natural sources including secondary metabolites of microorganisms [11-16]. These studies have gauged the nematicidal activity of candidates primarily by counting the number of live/dead nematodes under the microscope using behavioral changes as a main criterion. We argue that such approaches have two issues: 1) checking the effect of chemicals/biologics one-by-one using microscope is laborious and time-consuming; 2) behavioral changes of nematodes such as decrease/lack of movement or stiff/straightened body does not necessary mean that the nematode is dead or metabolically inactive, possibly leading to over-estimation of nematicidal activity. In order to address such issues, methodologies employing fluorescent dyes were developed to evaulate the viability of nematodes, particularly for testing new anthelminthic compounds. However, no such easy, economic, and timesaving techniques have been reported yet for the pine wilt nematode [17].

Here we describe the development of viability/mortality assay based on the use of redox dye that changes its color in response to degree of metabolic activity within the $B$. xylophilus population tested. Our method for quantitation of cell viability/mortality is independent of inspection of individual nematodes under microscope, enabling visual assessment of whole metabolic activities in the population. We demonstrate that our approach is applicable to rapidly and accurately testing effectiveness of chemicals or biologics against these deadly pine pathogens.

\section{Materials and Methods}

\section{Culture and Harvest of Bursaphelenchus xylophilus}

Bursaphelenchus xylophilus was obtained from the National Institute of Forest Science (NIFS, Korea) and used throughout this work. B.xylophilus was multiplied by growing them on PDA (potato dextrose agar) plate cultures having Botrytis cinerea, which was also obtained from NIFS. For harvest of the nematode, plate cultures were flooded with $25 \mathrm{ml}$ of sterile water and then were shaken 5 to 10 times to recover nematodes in a Falcon $50 \mathrm{ml}$ conical centrifuge tube. Using $20 \mu$ of B. xylophilus suspension, the number of nematodes was counted under a microscope.

\section{Setup of Colorimetric Assay Using Resazurin}

To determine the optimal number of nematodes and optimal dose of resazurin dye (Sigma-Aldrich, USA), we tested different combinations of nematode numbers and resazurin doses in 96well plates. The numbers of nematodes tested were 100, 200, and 500 . The amount of resazurin tested was 10,20 , and $30 \mu \mathrm{l}$. In all experiments, total volume was adjusted to $120 \mu \mathrm{l}$ in 96-well plates and nematodes were incubated with resazurin at $25^{\circ} \mathrm{C}$ and $70 \mathrm{rpm}$ for $16 \mathrm{~h}$. Color change was monitored at intervals of $4 \mathrm{~h}$ for 2 days in order to determine the timing of color change. To test if the colorimetric assay actually exhibits a range of color spectrum depending on the survival rate of nematode, we checked out color changes in mixtures of live and dead nematodes ranging from $100 \%$ survival to $0 \%$ survival on a 96 -well plate. The dead nematode was prepared by microwave for $1 \mathrm{~min}$ and mixed with live nematode proportions, following counting under microscope. Before incubation, absorbance was measured using a 96-well plate reader to determine if resazurin was actually reduced by metabolism of nematodes. Absorbance was measured at the wavelength of $630 \mathrm{~nm}$, at which absorbance difference between resazurin and resorufin in absorbance is the largest.

\section{Colorimetry-Based Assay on Drug Treatment}

To test applicability of the assay for screening of nematicidal chemicals, we used abamectin dissolved in methanol. In order to rule out the effect of methanol on nematode viability, colorimetric assays were first performed at $100 \%, 50 \%, 20 \%, 10 \%, 7 \%, 5 \%$, and $3 \%$ of methanol. Using information on solvent concentration, abamectin was added to the wells containing nematodes in amounts of $200,100,50,25,10,5$, and $1 \mu \mathrm{M}$ at final concentration.

\section{Colorimetry-Based Assay on Biologics Treatment}

In a previous study, a random insertional mutant library was prepared using EZ-Tn5 (Epicentre Technologies Corp., USA) mutagenesis kit in Raoultella ornithinolytica, which was isolated and shown to have strong nematicidal activity against B. xylophilus [18]. Mutant strains of $R$. ornithinolytica were cultured in 96-well plates using LB media. LB media were removed by spin-down of the plates, and then approximately 200 nematodes were added to each well along with $20 \mu \mathrm{l}$ of resazurin. A total volume of suspensions was set at $120 \mu \mathrm{l}$ and cultured for $16 \mathrm{~h}$ at $25^{\circ} \mathrm{C}$ and $70 \mathrm{rpm}$ for monitoring color changes.

\section{Results}

\section{Resazurin as a Redox Dye for Testing Cell Viability/ Mortality}

The assay we developed utilized a redox dye, resazurin (7-Hydroxy-3H-phenoxazin-3-one-10-oxide), which is used for measurement of metabolic activity and proliferation of living cells. In principle, the dye changes its color by bioreduction of oxidized form (blue) and concomitant increase of fluorescent intermediate (red) (Fig. 1). When we added nematodes to suspensions containing resazurin, we were indeed able to see color change, which corresponds to conversion of resazurin into resorufin through metabolic activity of nematodes (Fig. 1, circles at the bottom), suggesting the potential of resazurin as an indicator for cell viability/mortality assay. Absorption spectrums of the two 


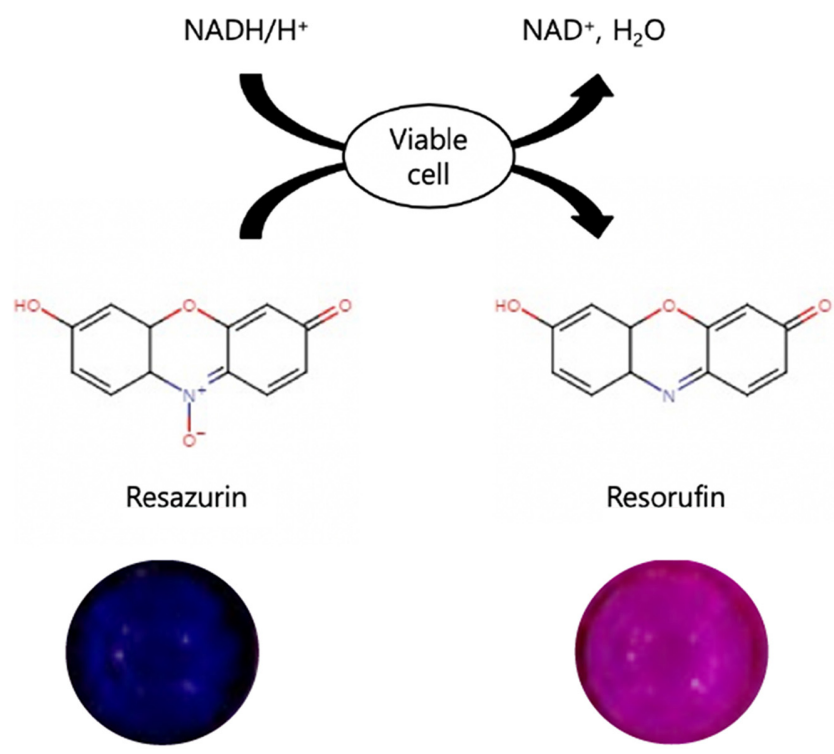

Fig. 1. Schemetic diagram showing conversion of resazurin into resorufin by metabolic activity of viable cells using NADH.

Metabolically active cells are able to change dye color from blue to pink depending on duration and intensity of cell metabolism.

forms show maximum difference in a range of wavelengths between 600 and $650 \mathrm{~nm}$. Therefore, it should be possible to monitor the remaining amount of resazurin by measuring absorbance at $630 \mathrm{~nm}$ wavelength.

\section{Correlation between Cell Viability/Mortality and Degree of Color Change}

Given the potential of resazurin as an indicator, we then tested sensitivity of color changes in measuring viability/ mortality rate. If the degree of color changes is not proportional to relative composition of live and dead nematodes in a population, our dye-based method would not yield quantitative data but only binary outcome (either alive or dead). In order to examine this, we generated nematode populations having different proportions of dead nematodes (Fig. 2A). In our experiment, nematode suspension was divided into two tubes, and dead nematodes were prepared by microwaving one tube for $1 \mathrm{~min}$ to kill them. Our preliminary experiment showed that one minute is the duration required to kill all the nematodes in the tube, while leaving them morphologically intact. When we placed nematode-containing suspensions from the microwaved tube onto Botrytis cinerea plate culture, which is used to feed nematodes for multiplication, no reduction in hyphal growth of $B$. cinerea was observed even after prolonged incubation, confirming $100 \%$ mortality by microwave. By mixing dead nematode suspensions with live ones, we obtained nematode populations having varying proportion of dead nematodes. Incubation following additions of resazurin to these populations showed a gradient of color changes that seems to roughly correspond to the portion of live nematodes (Fig. 2B). Further confirmation of such correlation was obtained by gradual decrease in absorbance measurement in response to increment in viability, indicating that degree of color changes is a relatively accurate reflection of nematode viability/mortality in the sample.

\section{Optimization of Colorimetric Assay}

Although color change of the redox dye is a good indicator for nematode viability/mortality, the read-out of the dye-based assay can vary depending on a number of
A

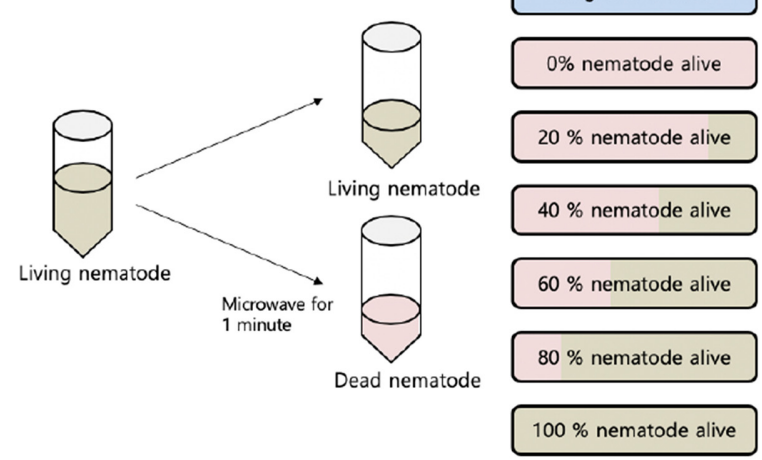

B

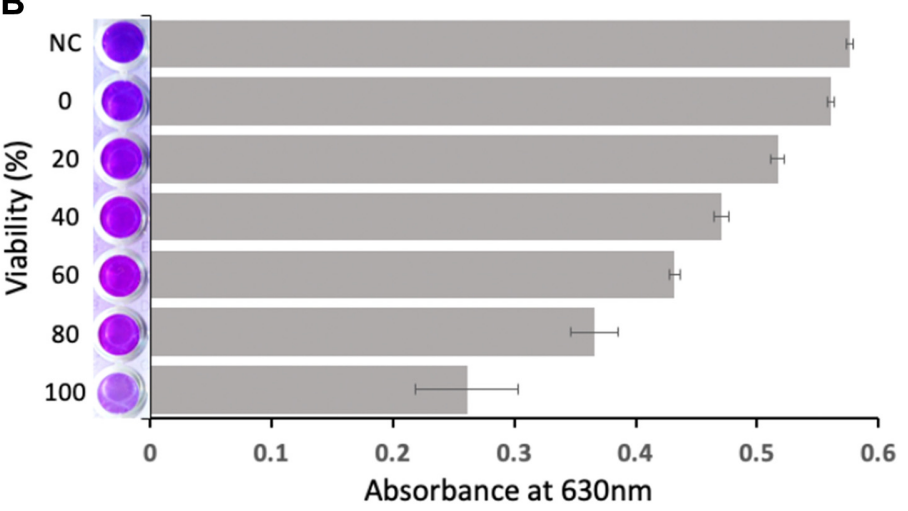

Fig. 2. Correlation between cell viability/mortality and degree of color change.

(A) Schematic diagram describing how populations of nematodes with varying proportions of dead nematodes were prepared. (B) Color changes and absorbance value at $630 \mathrm{~nm}$ for different population of nematodes. 
A

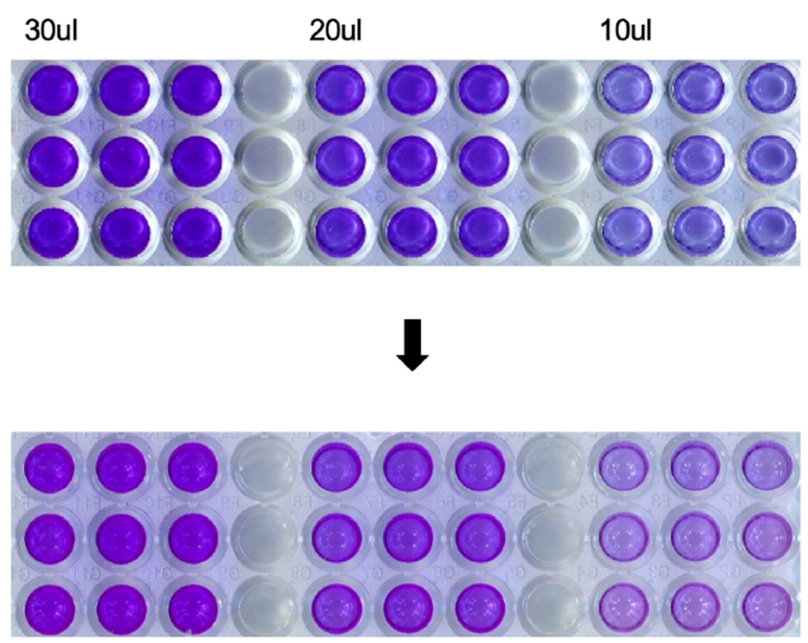

B

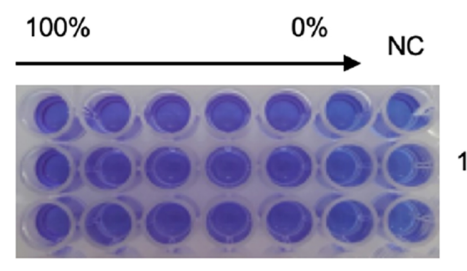

100unit

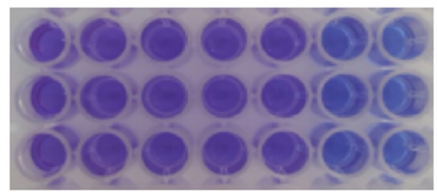

200unit

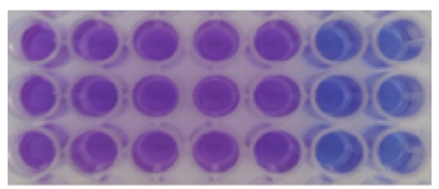

500unit

Fig. 3. Optimization of colorimetric assay parameters.

(A) Effects of dye quantities for optimal colorimetric assay. (B) Effect of number of nematodes in the wells for optimal colorimetric assay. Unit indicates the number of nematodes tested in each well, while percentage values indicate the proportion of dead nematodes among the total number of nematodes in the well ( $20 \%$ interval). NC indicates negative control (no nematodes added to the well).

parameters including incubation time, amount of dye and number of nematodes used. To find the optimal parameter settings that can maximize the range of gradient in color change at read-out, we ran a series of experiments in which we changed the amount of dye and number of nematodes. We first tested effects of resazurin dose on assay outcome. Using different amounts of resazurin on a fixed number of nematodes ( 200 nematodes), we found that using $20 \mu \mathrm{l}$ of dye allows the largest contrast in color change after $16 \mathrm{~h}$ of incubation (Fig. 3A). Using less $(10 \mu \mathrm{l})$ or more $(30 \mu \mathrm{l})$ dye resulted in much smaller range of gradient.

Next, we examined the effect that the number of nematodes has on the assay by letting the number vary from 500 through 200 to 100 with fixed incubation time of $16 \mathrm{~h}$. Our data showed that if we use 500 nematodes in each well, color change occurs rapidly regardless of proportion
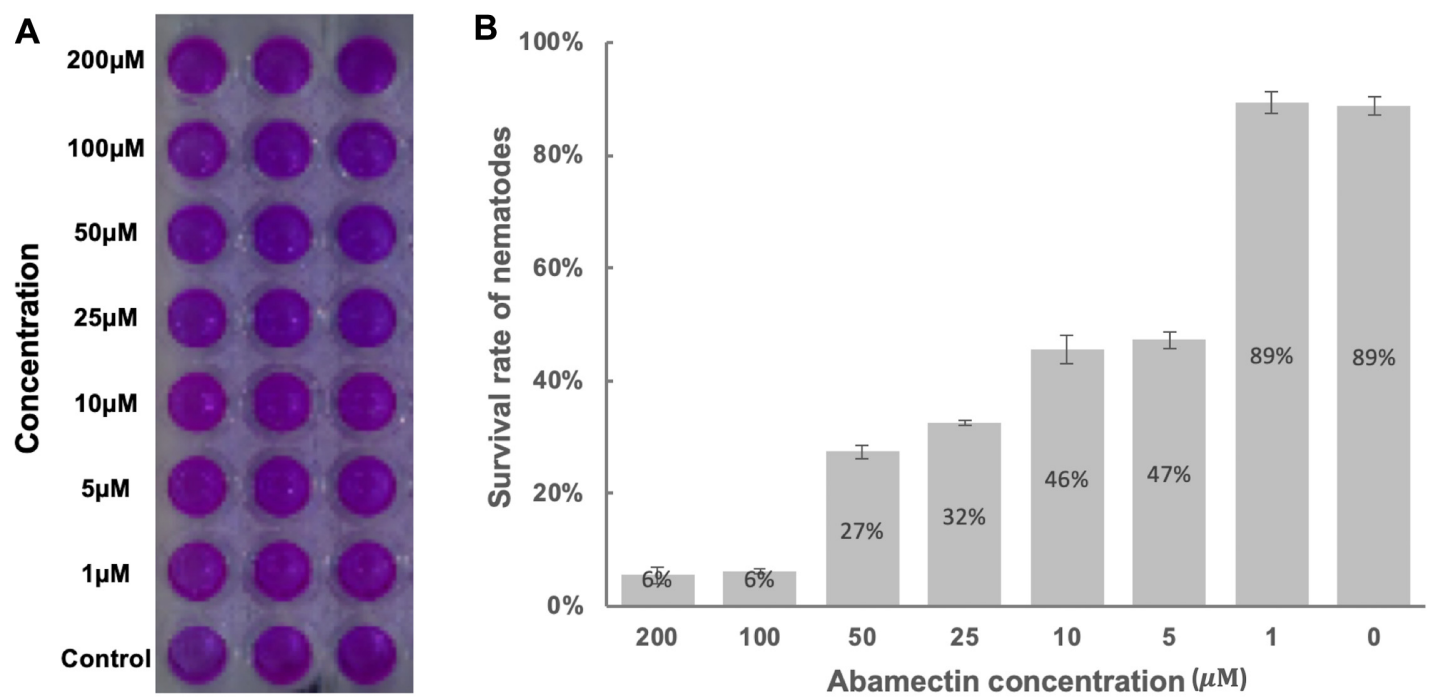

Fig. 4. Application of colorimetric assay for testing efficacy of abamectin.

(A) Color changes in samples treated with various concentration of abamectin. (B) Visual evaluation of nematode viability/mortality for comparison to color changes in (A). 
of dead nematodes (Fig. 3B). On the contrary, it was difficult to differentiate among samples having different portion of dead nematodes, when only 100 nematodes were used for the assay. Under our experimental condition, use of 200 nematodes and $20 \mu \mathrm{l}$ of dye with $16 \mathrm{~h}$ incubation provided us with the largest contrast among samples. It should be noted that we did not test incubation time independently, since optimal incubation time is dependent on other variables.

\section{Test for Nematicidal Activity of Abamectin}

Following optimization, we asked if our assay could be applied to evaluation of chemicals for their nematicidal activities. Since a library of chemicals is not available to us, we chose to use the well-known nematicidal compound, abamectin to test whether our assay is able to assess the nematicidal activity of abamectin with enough sensitivity. Our results showed that abamectin could kill nearly all the nematodes in our sample at $100 \mu \mathrm{M}$ or higher concentration. Interestingly, however, color change occurs as the dose of abamectin added to nematode suspensions increases, although it does not appear to be gradual (Fig. 4A). Rather, change seemed to occur only after particular concentration is reached. When we counted the number of live/dead nematodes in the same samples under the microscope, plotting the data indeed showed a staircase pattern, corroborating our observation on color changes (Fig. 4B). Again, these results indicate that our assay method using redox dye is able to accurately measure nematicidal activity of candidate compounds.

\section{Test for Screening of Mutant Library}

In a previous study, it was shown that an endophytic bacterium, Raoultella ornithinolytica, which was isolated from plants, possesses strong activity against B. xylophilus [18]. In an effort to identify genes or pathways responsible for such nematicidal activity, an insertional mutant library $(n=1,343)$ was generated for $R$. ornithinolytica in our lab using Tn5 mutagenesis method. Such resource allowed us to test if we could scale up our assay for high throughput screening. Using the wild-type strain as a positive control, we screened all the mutants. In our screening, we used $6 \mathrm{~h}$ of incubation instead of $16 \mathrm{~h}$. This is because the bacterium itself would cause changes in dye color through its own metabolic activity. However, such color change will be accelerated by live nematode if the mutant loses the ability to kill them. Therefore, the rationale behind our screening scheme is that any mutants, in which key nematicidal genes are disrupted, would be impaired in biosynthesis of

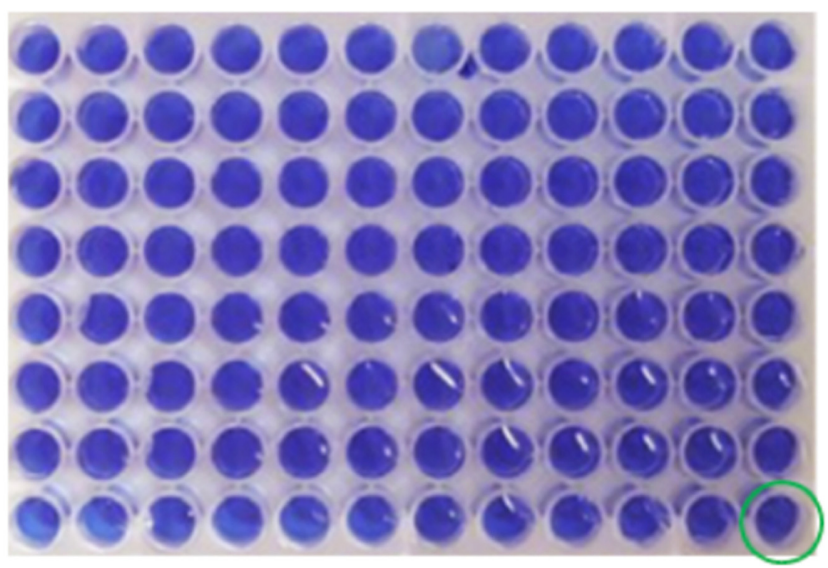

\section{$6 h$}

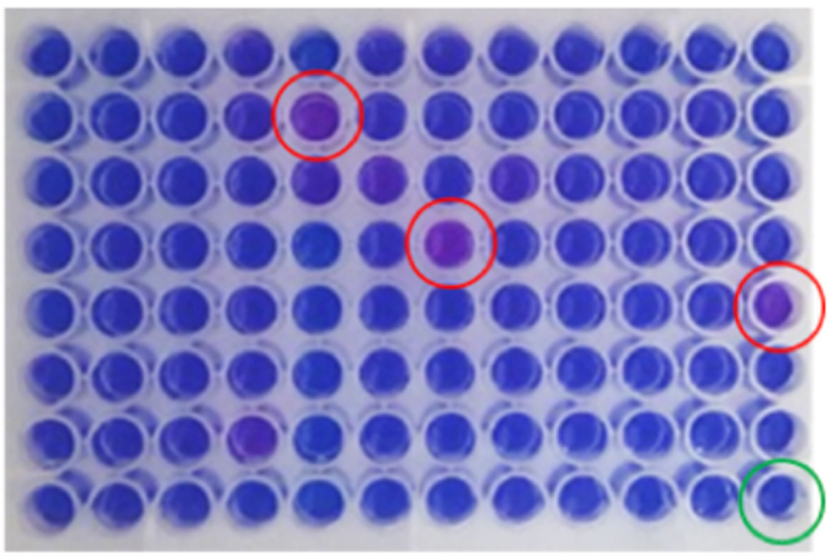

Fig. 5. Representative image of plates showing color changes in response to treatment of nematodes with biologics derived from R. ornithinolytica Tn5 mutant library.

Wild-type strain of $R$. ornithinolytica (positive control) was marked with green circle. Mutant strains showing no/reduced nematicidal activity are marked with red circles.

nematicidal compound(s) and consequently result in color change of dye much faster than the wild-type strain. Through the screens using our redox dye-based assay, a single person was able to identify 27 candidate mutants warranting further genetic studies within three days, indicating the scalability of our assay method (Fig. 5).

\section{Discussion}

In this work, we have developed a colorimetric assay based on the use of redox dye resazurin for rapid and quantitative assessment of viability/mortality of PWN. In 
efforts to discover alternatives to chemicals such as abamectin that have been heavily used for control of PWD, screening a large number of candidate chemicals/biologics can be one of the constraints. Conventional method for evaluating viability/mortality of nematodes relies on visual inspection and numeration of individual nematodes in the sample. Such a method is laborious and timeconsuming. Moreover, judging viability of nematodes using visual and behavioral criteria can be mis-leading, resulting in over-estimation of mortality. One remedy for the problem is to use fluorescent dye, of which uptake into the cell is dependent on the functions and integrity of cellular membranes [17]. However, one still has to check individual nematodes one-by-one down the microscope, and determining viability/mortality based on fluorescence staining patterns can be ambiguous, making it impractical to use for high throughput screening.

On the contrary, methods using redox dye such as resazurin do not require the use of microscope and are able to easily quantify viability/mortality for a large number of samples just by monitoring color changes and/or measuring absorbance at a particular wavelength. Assumptions underlying the redox dye-mediated assay are mainly twofold: 1) the amount of color change should be proportional to the viability of nematodes in the sample; 2) viability/ mortality of nematodes should be a single factor affecting redox dye chemistry. In our work, we showed that degree of color change (or absorbance measurements) correlates linearly to the proportion of live nematodes in the sample, indicating that our assay condition using resazurin meets the first assumption. The second assumption is related to the solvent used to dissolve candidate chemicals or media used to culture biologics. The LB medium we used in our assay affected neither viability/mortality of B. xylophilus nor chemistry of resazurin. However, we found that the methanol, which is used to dissolve abamectin, had some influence on color changes, although it did not signficantly change the viability/mortality of nematodes at a low concentration (but high enough to dissolve abamectin), suggesting that choice of solvents and their concentration can be important factors. Therefore, if a number of candidate nematicides needs to be dissolved in different solvents, extreme caution is required to obtain correct readout from the use of our assay.

In summary, our results demonstrated that the redox dye-based colorimetric assay developed here is easily scalable and applicable to screening of candidate chemicals and biologics. We anticipate that our assay would facilitate rapid discovery of chemicals and biologics from a large number of candidates. It is also possible to apply our assay to studies aiming at providing information regarding the molecular and cellular basis for pathogenesis mechanisms of B. xylophilus. For example, our assay may serve as a platform technology for lifespan, biotic, and abiotic stress resistance assays. Screening of B. xylophilus mutants in particular would enable identification of key genes and their protein products that can be targeted for development of chemicals having potential to inhibit growth and development of this deadly pathogen.

\section{Acknowledgments}

This work was supported by a grant from the National Institute of Forest Science (Project No. FE0702-2016-02-2016).

\section{Conflict of Interest}

The authors have no financial conflicts of interest to declare.

\section{References}

1. Mota MM, Vieira P. 2008. Pine wilt disease: a worldwide threat to forest ecosystems, pp. 1-14. Springer, Heidelberg, Germany.

2. Yano S. 1913. Investigation on pine death in Nagasaki prefecture. Sanrin-Kouhou. 4: 1-14.

3. Steiner G, Buhrer EM. 1932. Aphelenchoides xylophilus, a nematode associated with blue-stain and other fungi in timber. J. Agric. Res. 48: 949-951.

4. Evans HF, McNamara DG, Braasch H, Chadoeuf J, Magnusson C. 1996. Pest risk analysis (PRA) for the territories of the European Union (as PRA area) on Bursaphelenchus xylophilus and tis vector in the genus Monochamus. EPPO Bulletin. 26: 199-249.

5. Jikumaru S, Togashi K. 2001. Transmission of Bursaphelenchus mucronatus (Nematoda : Aphelenchoididae) through feeding wounds by Monochamus saltuarius (Coleoptera : Cerambycidae). Nematology 3: 325-333.

6. Mota MM, Braasch H, Bravo MA, Penas AC, Burgermeister W, Metge K, et al. 1999. First report of Bursaphelenchus xylophilus in Portugal and in Europe. Nematology 1: 727-734.

7. Mamiya Y. 1988. History of pine wilt disease in Japan. J. Nematol. 20: 219-226.

8. Mamiya Y. 1983. Pathology of the pine wilt disease caused by Bursaphelenchus xylophilus. Annu. Rev. Phytopathol. 21: 201-220.

9. Futai K. 2013. Pine wood nematode, Bursaphelenchus xylophilus. Annu. Rev. Phytopathol. 51: 61-83.

10. Kwon TS, Song MY, Shin SC, Park YS. 2005. Effects of aerial insecticide sprays on ant communities to control pine wilt 
disease in Korean pine forests. Appl. Entomol. Zool. 40: 563574.

11. Rajasekharan SK, Lee JH, Ravichandran V, Lee J. 2017. Assessments of iodoindoles and abamectin as inducers of methuosis in pinewood nematode, Bursaphelenchus xylophilus. Sci. Rep. 7: 6803

12. Kim J, Lee SM, Park CG. 2016. Bursaphelenchus xylophilus is killed by homologues of 2-(1-undecyloxy)-1-ethanol. Sci. Rep. 6: 29300

13. Shanmugam G, Lee SK, Jeon J. 2018. Identification of potential nematicidal compounds against the pine wood nematode, Bursaphelenchus xylophilus through an In Silico Approach. Molecules 23: 1828

14. Lee HR, Lee SC, Lee JE, Seo SM, Jeong YC, Jung CS, et al. 2017. Nematicidal activity of 3-acyltetramic acid analogues against pine wood nematode, Bursaphelenchus xylophilus. Molecules 22: 1568
15. Seo SM, Kim J, Koh SH, Ahn YJ, Park IK. 2014. Nematicidal activity of natural ester compounds and their analogues against pine wood nematode, Bursaphelenchus xylophilus. J. Agr. Food Chem. 62: 9103-9108.

16. Liu MJ, Hwang BS, Jin CZ, Li WJ, Park DJ, Seo ST, et al. 2018. Screening, isolation and evaluation of a nematicidal compound from actinomycetes against the pine wood nematode, Bursaphelenchus xylophilus. Pest Manag. Sci. 75: 1585-1593.

17. Ferreira SR, Mendes TA, Bueno LL, de Araujo JV, Bartholomeu DC, Fujiwara RT. 2015. A new methodology for evaluation of nematode viability. Biomed. Res. Int. 2015: 879263.

18. Shanmugam G, Dubey A, Ponpandian LN, Rim SO, Seo ST, Bae $\mathrm{H}$, et al. 2018. Genomic insights into nematicidal activity of a bacterial endophyte, Raoultella ornithinolytica MG against pine wilt nematode. Plant Pathol. J. 34: 250-255. 